\begin{abstract}
Paolo Branca
(professore associato di Lingua e letteratura araba nell'Università Cattolica di Milano, Dipartimento di Scienze Religiose, Facoltà di Lettere e Filosofia)
\end{abstract}

\title{
Corano e/o Sunna? \\ Funzione narrativa e prescrittiva nelle 'fonti' dell'Islam ${ }^{1}$
}

\section{Quran and/or Sunna? Narrative and prescriptive function in the 'sources' of Islam}

\begin{abstract}
Even if in different forms and according to historical and anthropological factors that have conditioned their development, religions have a doctrinal part and precepts that can have different weight and priorities in each of them. Islam, which like Judaism is centered more on orthopraxis than on orthodoxy, over time and especially in the modern era, also in relation to the phenomenon of huge migrations of its faithful to the West, is faced with new challenges affecting its regulatory side. In this regard, various orientations and studies have long confronted each other and still animate a little-known debate outside the circle of specialists.
\end{abstract}

SOMMARIO: 1. Prologo - 2. A mo' d'introduzione - 3. Storia delle origini od origini di una storia? - 4. Fra' tacere' e 'dire', fra 'ordinare' e 'interdire' ... - 5. Rottura di un equilibrio - 6. Emanciparsi dalla Sharî‘a o applicarla? La disputa fra Corano e Sunna - 7. Solo il Corano basta - 8. La critica degli orientalisti - 9. Ulteriori studi: ICMA (isnad-cum-matn analysis)- 10. Maqâssid al-Sharî‘ $a$ (le finalità della legge islamica) - 11. Stimoli e riflessioni da un tentativo di approccio comparativo - 12. Una possibile 'inversione' di tendenza? - 13. Con il tempo e con la paglia ...

\section{1 - Prologo}

Le recenti disposizioni del Ministero per la promozione della virtù e l'interdizione del vizio del neo-costituito Emirato Islamico dell'Afghanistan, sotto la guida dei Talebani, a proposito del permesso accordato a una donna musulmana non accompagnata di compiere un viaggio entro una determinata distanza, hanno risvegliato l'interesse circa una questione da sempre presente nelle norme della Sharî‘a, tutt’ora oggetto di dibattito.

\footnotetext{
${ }^{1}$ Contributo sottoposto a valutazione. Article peer evaluated.
} 
Fatwa n. 88562 del 5 settembre 2004

Domanda

Quando una donna non ha marito - o è vedova o divorziata e non ha altri parenti Mahram² viventi - quali sono le regole per lei riguardo a un viaggio minore di un giorno e una notte (va calcolato il tempo totale del viaggio da casa sua? la destinazione deve comportare uno spostamento inferiore un giorno e una notte?) e quando viaggia per più di un giorno e una notte? Ha bisogno di un Mahram? Inoltre, come si misura il viaggio quando vuole spostarsi dal suo luogo di residenza ad un altro luogo, ma si ferma per una tappa intermedia per meno di un giorno e una notte? E se si ferma per più di tale intervallo? Ha bisogno di un Mahram in qualsiasi momento?

Risposta

La lode perfetta appartiene a Iddio, Signore dell'universo. Attesto che non c'è nessuno degno di adorazione tranne che Iddio e che Muhammad è il Suo Servo e Messaggero. Chiediamo a Iddio che siano sempre esaltati il nome del Profeta, della sua famiglia e di tutti i suoi compagni.

Sembra che tutte le tue domande riguardino la distanza che una donna può percorrere senza un Mahram (un parente con cui non possa contrarre matrimonio). Le narrazioni riportate nei libri di alBukhari, Muslim e di altri a questo proposito differiscono. Una recita: "Una donna non dovrebbe viaggiare per una distanza di tre giorni se non con un Mahram". Un'altra narrazione recita: "Non è permesso per una donna che crede in Dio e nell'Ultimo Giorno viaggiare per una distanza di un giorno e una notte senza un Mahram".

Un'altra ancora vieta a una donna di viaggiare senza Mahram, ma non specifica né tempo né distanza:

"Una donna non dovrebbe viaggiare se non con un Mahram".

"Tuttavia, un'altra narrazione specifica che la distanza è di 12 miglia. Se ne evince che non si intende la distanza, ma il viaggio in se stesso. Pertanto non è permesso a una donna percorrere una distanza che è considerata un viaggio secondo le usanze del suo paese senza un Mahram. Questa è l'opinione prevalente. Non c'è differenza se il tragitto è continuo o interrotto da pause. A ogni modo, se una donna è obbligata a viaggiare e non ha trovato un Mahram o il marito che l'accompagni, allora Iddio non glielo impedisce perché le necessità rendono permesso ciò che è proibito. Dice infatti il Corano: "Iddio non ha imposto alcuna difficoltà nella religione (22:78), e un bisogno

2 Per i termini arabi abbiamo adottato una traslitterazione semplificata che indica soltanto le vocali lunghe senza ricorrere a punti diacritici o altri grafemi poco noti ai non specialisti. 
urgente è considerato una necessità. Ma Iddio lo sa meglio"3.

Nonostante possa apparire paradossale, l'episodio rivela un problema irrisolto che si ripropone in varie consultazioni che a stampa o nel web ricorrono più di quanto si pensi:

"Se mentre sto pregando per strada mi si sovrappone l'ombra di una donna (che potrebbe essere mestruata) o quella di un cane (animale impuro) la mia preghiera resta valida?"; "Se andando in motorino durante Ramadan ingoio involontariamente un moschino, il giorno di digiuno resta valido o lo devo recuperare alla fine del mese?".

Sono domande che possono far sorridere, ma rivelano la preoccupazione di molti fedeli musulmani circa la validità dei loro atti di culto, come del resto si riscontra in Israele dove gli ascensori nel giorno di sabato di fermano a ogni piano, per evitare agli utenti di premere il tasto del piano desiderato per non attivare un contatto elettrico paragonabile all'accensione di un fuoco, interdetta dalla Torà nel giorno del riposo settimanale o dove è diffuso l'utilizzo di timer programmati per l'accensione di luci o apparecchiature varie di sabato.

\section{2 - A mo' d'introduzione}

La religiosità precede la religione, così come il linguaggio orale precede ogni scrittura. In quest'ultima si ha una sorta di istituzionalizzazione graduale che fissa le altrimenti fluide (ma non assenti) regole e strutture del parlato.

Così come ognuno di noi, crescendo, rielabora e in parte dimentica o comunque riduce il peso del rapporto simbiotico con la madre, anche le religioni - istituzionalizzandosi - assumono un aspetto più prescrittivo e normativo (di stampo più paterno che materno), più etico che estetico, funzionale a uno dei ruoli che da un lato si trovano a svolgere e dall'altro ci si attende da esse.

Il carattere eminentemente narrativo delle Scritture, non solo nelle fedi 'rivelate', cede progressivamente il passo a quella pedagogica, finalizzata a un progetto tanto inevitabile quanto inevitabilmente solo parziale.

3 https://www.islamweb.net/en/fatwa/88562/the-travelling-woman-who-has-no-mahram-to-es cort-her 
Lo dimostra, fra l'altro, la contraddizione intrinseca di una rivelazione che riteniamo completa, ma che è al tempo stesso, per propria ammissione e in forme iperboliche, inesauribile:

"Vi sono ancora molte altre cose compiute da Gesù, che, se fossero scritte una per una, penso che il mondo stesso non basterebbe a contenere i libri che si dovrebbero scrivere" (Gv. 12, 25); «Di': "Se il mare fosse inchiostro per scrivere le Parole del mio Signore, di certo si esaurirebbe prima che fossero esaurite le Parole del mio Signore, anche se Noi ne aggiungessimo altrettanto a rinforzo"» (Corano, 18, 109).

\section{3 - Storia delle origini od origini di una storia?}

Un autore latino, che si esprimeva in greco, ai tempi dell'imperatore Giuliano l'Apostata, ebbe a scrivere con grande intuito: "queste cose non furono mai, ma sono sempre" indicando il valore simbolico delle divinità e dei miti antichi. Non ebbe successo, naturalmente, poiché il cambiamento di paradigma era ormai avviato e inarrestabile, ma la sua intuizione non dovrebbe essere per questo sottovalutata ${ }^{4}$.

Non solo la modernità, ma anche altri approcci filosofici precedenti, hanno moltiplicato le fratture, specialmente fra spirito e materia, a vantaggio prima di uno dei due corni del dilemma, poi del secondo, fino alla vera e propria sbornia tecnologica del tempo presente.

Non sarebbe male, dunque, recuperare il senso non tanto spiritualistico (oppositivo) quanto quello olistico dell'esperienza religiosa che originariamente 'non' è esperienza solo di 'altro', ma prima e soprattutto esperienza della totalità dell'essere.

Indubbiamente le religioni comportano anche un discrimine... quasi un principio d'esclusione: tra luce e tenebre, tra bene e male, tra chi accetta il messaggio e chi lo rifiuta, tra chi rispetta determinati divieti e precetti e chi non lo fa.

Ma dallo stesso messaggio siamo messi in guardia rispetto a troppo automatiche e trancianti opposizioni:

"I servi del Misericordioso sono coloro che camminano umilmente sulla terra e quando un ignorante si rivolge a loro rispondono 'Pace!'" (Corano 25, 63).

"Se Dio avesse voluto avrebbe fatto di voi una comunità unica,

${ }^{4}$ Cfr. SAlUSTIO, Sugli dèi e il mondo, a cura di R. Di GIUSEPPE, Adelphi, Milano, 2000. 
ma così non ha fatto per mettervi alla prova in ciò che vi ha donato. Gareggiate dunque nelle opere buone, a Dio tutti farete ritorno ed Egli allora vi informerà sulle cose in cui siete stati in discordia" (Corano, 5, 48).

Quando, in qualsiasi fede, vengono sottolineate eccessivamente fratture nello stesso individuo, tra lui e il suo ambiente e i suoi simili o con il suo stesso Creatore, qualcosa d'essenziale di una genuina esperienza religiosa viene a mancare con gravi conseguenze.

Una logica binaria va bene per le macchine, non per gli esseri umani: ricordo una giovane musulmana che paragonava il Corano al libretto d'istruzioni di una lavatrice, pretendendo che solo chi ha fabbricato sia $i$ tessuti sia la macchina possa indicarne il buon funzionamento.

Dobbiamo dunque chiederci se nelle fonti e nelle esperienze dei seguaci delle varie religioni sussistano elementi in grado di andar oltre una simile disperante prospettiva che nulla aggiunge, ma molto anzi riduce, rispetto a un'origine e a una permanenza comunque testimoniate:

"Drizza quindi il tuo volto alla vera Religione, in purità di fede, Natura prima (fitra) in cui Dio ha naturato gli uomini. Nessun mutamento patisce la Creazione di Dio: quella è la Religione retta, ma i più degli uomini non sanno" (Corano, 30,30)

Raggruppamenti o movimenti puritani e intransigenti all'interno delle varie tradizioni religiose ve ne sono più o meno sempre stati, ma 'integralismo' e 'fondamentalismo' sono fenomeni moderni tanto nella loro genesi quanto nelle loro dinamiche.

Essi non pongono tanto la distinzione tra credenti e non, ma fra tradizione e modernità: per questo non si possono comprendere con un approccio unicamente dottrinale o teologico. Non aderiscono a un credo diverso da quello degli altri fedeli 'comuni', ma hanno una maniera particolare di 'appartenere' alla comunità dei credenti. $\mathrm{O}$, meglio, non tanto alla comunità religiosa storica che nel tempo e nello spazio ha assunto differenti forme, ma a un 'noi' che si presume la mera attualizzazione dell'unico, immutabile e perfetto modello delle origini. Preservazione di un mito che però non corrisponde ad alcuna realtà effettiva di nessun tempo e in nessun luogo.

La straordinaria fioritura dell'aspetto normativo nell'Ebraismo e nell'Islam, considerate giustamente delle ortoprassi piuttosto che delle ortodossie, può aver avuto fra le sue cause il fatto che all'atto genetico e fondativo delle due religioni i corrispondenti popoli non avevano che consuetudini tribali, poco dettagliate e difformi, per cui i 'fondatori' e le 
'rivelazioni' si trovarono nella necessità di colmare tale vuoto. Non così andò per il Cristianesimo che si trovò ben presto a istituzionalizzarsi avendo nel retroterra delle zone in cui andava diffondendosi maggiormente, eredità complesse e strutturate quali il pensiero greco e il diritto romano.

Per quanto riguarda l'influsso di altri popoli e tradizioni se nell'Ebraismo hanno avuto un ruolo importante, tra altri e prima di essi, gli antichi Egizi e i Babilonesi, per l'Islam delle origini i modelli persiano e bizantino prevalsero, mentre per il Cristianesimo, dopo la prima fase ellenistica e romana, non di scarso impatto sarebbe stato anche l'influsso barbarico che, con Franchi e Germani, avrebbe prolungato un 'Sacro' Romano Impero fino alla Prima Guerra mondiale.

\section{4 - Fra 'tacere' e 'dire', fra 'ordinare' e 'interdire' ...}

Per quanto considerate 'sacre' e 'rivelate', le Scritture non pretendono dunque di dire tutto, ma qualcosa che col 'tutto' ha a che fare, intendendo essenzialmente con ciò il principio e la fine e quindi il senso del creato e delle creature ... Del resto, lo stesso linguaggio umano (o comunque comprensibile dagli esseri umani) in cui sono espresse condivide gli stessi limiti e le medesime caratteristiche: non tutto ha un nome (ad esempio, le infinite gradazioni e tonalità dei colori) poiché sarebbe antieconomico, ma allo stesso tempo le strutture di base del linguaggio e quindi del pensiero rivelano substrati nascosti e talvolta inconsci del nostro essere.

Un noto detto popolare afferma: 'chi tace acconsente', ipotizzando un'approvazione $e$ silentio di quanto non normato, ma è evidentemente una soluzione di buon senso che non può esser sempre ritenuta valida $\mathrm{e}$ che svela il bisogno di risposte evidentemente assenti o non esplicite in qualcosa che se da un lato ha anche un valore e una funzione normativi, non per questo può essere considerato un completo codice morale e comportamentale tout-court.

Nel caso dell'Islam si è posta ben presto la necessità di dare agli usi e alle prassi locali di quel che rapidamente si stava trasformando in un vasto impero, un fondamento e una sistematicità che almeno non contrastassero coi principii generali enunciati dal Corano, anche a motivo della scarsità di versetti prettamente giuridici (poche centinaia su oltre seimila), della mancanza di molti dettagli anche sugli articoli del credo e i precetti del culto, della presenza di disposizioni contrastanti o che si prestavano a differenti interpretazioni e via dicendo. 
Venne in soccorso la Sunna ${ }^{5}$, termine che indicava già presso gli arabi antichi gli usi e i costumi cui si uniformavano i membri delle varie tribù nel loro comportamento, fondati sui valori degli antenati. Il concetto di tradizione permane anche nell'accezione islamica della stessa parola, ma assume un significato più specifico. Nel Corano, infatti, il vocabolo indica principalmente il modo di agire tenuto da Dio verso i popoli ai quali, nel corso dei secoli, ha mandato i propri profeti e inviati; successivamente il termine si è specializzato e con Sunna si è venuta a indicare anzitutto la consuetudine del Profeta e dei suoi primi Compagni, destinata ad assumere col tempo un valore normativo sempre maggiore. Fu così che gli insegnamenti, le azioni e gli stessi silenzi con cui il Profeta offriva un tacito assenso a quanto altri compivano sotto i suoi occhi divennero altrettanti precedenti sui quali fondare la legittimità della condotta dei musulmani nelle più varie situazioni. I racconti che riportano i fatti in questione sono detti in arabo hadîth, termine che significa originariamente "detto" o "fatto". In ogni hadith, il testo (matn) del racconto è preceduto da una lista di nomi, detta isnâd, ossia "sostegno", che elenca la serie di quanti si sono tramandati il racconto nel corso del tempo.

Così come era avvenuto per il Corano, la spinta decisiva per il passaggio dalla forma orale a quella scritta venne anche per la Sunna dalla progressiva scomparsa di quanti ne erano stati testimoni diretti. Nacquero così le prime raccolte, seguite da altre sempre più voluminose, ove i hadith erano raggruppati in base a svariati criteri: talvolta prevaleva il tema trattato, altre volte il nome del più antico trasmettitore e così via...

Vari gruppi fecero in modo di far confluire nella Sunna espressioni attribuite al Profeta favorevoli ai propri interessi e ciò metteva potenzialmente in pericolo la sua stessa credibilità, così alcuni specialisti sottoposero ad attenta analisi una mole impressionante di hadîth, riunendo quelli più sicuri in apposite raccolte che hanno costituito la base della giurisprudenza islamica. La loro opera di verifica si basò, più che su criteri contenutistici e formali, sull'attendibilità della catena dei trasmettitori. I hadîth passati al vaglio confluirono nei cosiddetti "Cinque Libri": i due Sahîh (ossia "Libro genuino") di al-Buhârî (m. 870) e Muslim (m. 875), le Sunan (ossia "Tradizioni") di Abû Dâ'ûd (m. 889), al-Tirmidhî (m. 893) e alNasâ'î (m. 915), cui se ne aggiunge talvolta un sesto.

Nella Sunna è confluita una grande quantità di materiali che trattano di una straordinaria varietà di temi, legati non soltanto al culto,

5 Cfr. J. BURTON, An Introduction to the Hadith, Edimburgh University Press, Edimburgh, 1994. 
ma anche a transazioni commerciali, disposizioni penali, regole di educazione ... La funzione di questi testi divenne di fondamentale importanza per l'elaborazione del diritto musulmano il quale è forse il frutto più caratteristico ed elaborato dell'Islam, religione poco incline a complesse formulazioni dogmatiche e più attenta alle disposizioni giuridiche e comportamentali.

Accanto alle due fonti scritte (Corano e Sunna), il diritto musulmano si basa anche sul consenso (ijmâ') della comunità dei credenti che però, per quanto attiene alle questioni più tecniche, si riduce ovviamente al consenso degli esperti in materia giuridica.

Queste prime tre fonti del diritto musulmano trovano nell'intervento divino la loro origine o comunque una garanzia di autenticità, la quarta fonte si basa invece soltanto sull'attività umana e pertanto è quella che ha fatto maggiormente discutere. Si tratta del principio di analogia (qiyâs) col quale per induzione, prendendo le mosse da casi simili regolati in base alle altre fonti del diritto, il singolo giurista (faqîh) giunge con il proprio criterio $\left(r a^{\prime} y\right)$ a desumere regole per situazioni inedite.

I più rigidi custodi della tradizione si opposero all'indiscriminata applicazione del qiyâs, che fu comunque seguito da molti giuristi e in particolare da quelli appartenenti alla più antica delle scuole sunnite, detta hanafita, dal nome di Abû Hanîfa (m. 767), ritenutone il fondatore. Il suo metodo si caratterizza per l'ampio ricorso alla valutazione personale del giurista, quando i testi non contenevano disposizioni sufficienti, per la considerazione riservata alla consuetudine ('urf) e a valutazioni di opportunità. Diffusasi inizialmente soprattutto in Iraq, la scuola si radicò presto in tutta l'area iranica, ma non mancarono dottori hanafiti anche nella parte occidentale dell'impero. Con gli Ottomani infine divenne scuola ufficiale, sebbene non esclusiva, e ancor oggi è quella che ha il maggior seguito nel mondo musulmano: è diffusa in Turchia, Asia Centrale, Afghanistan, India e Pakistan.

Legato alla tradizione medinese fu invece Malik ibn Anas (m. 795), iniziatore della scuola da lui poi detta appunto malikita. I suoi responsi (fatâwâ, plurale di fatwâa) hanno un solido fondamento nella Sunna, ma non manca il ricorso al qiyâs e l'utilizzo di criteri sussidiari come la valutazione del bene comune. Anche l'insegnamento di Malik trovò molte adesioni e si diffuse soprattutto nelle province occidentali dell'impero e ancor oggi prevale nel Maghreb.

Chi diede un contributo fondamentale alla sistematizzazione della disciplina fu Muhammad al-Shâfi î (m. 820), iniziatore della scuola da lui detta shafi'ita. Il suo merito fu quello di precisare esplicitamente e di 
sistematizzare le fonti del diritto musulmano (usûl al-figh). Rispetto agli hanafiti egli ridusse l'ampiezza del ricorso alla valutazione del singolo giurista, dando maggiore importanza alla Sunna, di quest'ultima però ritenne vincolanti soltanto le parti risalenti direttamente al Profeta, mentre allargò il ricorso al "consenso" riferendolo all'insieme di tutti i dotti in materia, senza ridurlo a quello dei soli appartenenti a una determinata scuola o di un certo territorio.

"It is in his discussion of the sunna and tradition that we find one of Shafi'i greatest contributions to Islamic jurisprudence. For although the school of tradition laid sufficient emphasis on the sunna, in opposition to the school of opinion, in which Safi'i himself tokk an active part his in early career [...]. Shafi'i made a distinction between an aythentic tradition of the Prophet and a narrative which embodies the opinion of a Companion or a leading jurist; the latter may be useful in clarifying the meaning of a text, but in should not be as binding as a tradition from the Prophet" ${ }^{\prime \prime}$.

Dopo il traumatico passaggio dalla dinastia Omayyade di Damasco (750) a quella Abbaside che si fisserà a Baghdad, il perdurare del conflitto sunniti-sciiti, il sorgere di diverse correnti dottrinali, l'influsso greco nella filosofia e l'esoterismo dei primi gruppi sufi, l'irrigidimento dovuto a Shafi'i ci pare più che altro un tentativo di impedire che anche in campo giuridico proliferassero in base agli usi locali e alle opinioni degli esperti di varia tendenza, normative troppo differenziate che mettessero a repentaglio la situazione di coesione e unità già problematica al primo sorgere della Umma.

Bahrain, Yemen, Indonesia e Africa orientale figurano tra le regioni dove gli sciafi'iti sono oggi maggiormente presenti.

La più rigida delle quattro scuole di diritto sunnite è quella che si riallaccia agli insegnamenti di Ahmad ibn Hanbal (m. 855) e detta quindi hanbalita. Così come in campo teologico egli era stato il grande oppositore dei razionalisti, in quello giuridico combatté il ricorso al ragionamento personale facendosi strenuo difensore dell'assoluta supremazia dei testi. Tra le fonti del diritto rifiutò pertanto il qiyâs. Il suo rigorismo guadagnò alla scuola hanbalita molte simpatie, specialmente nelle regioni centrali dell'impero islamico. Con l'avvento degli Ottomani essa fu relegata soprattutto nella penisola araba e oggi ha la sua roccaforte in Arabia Saudita, fondata sulla dottrina puritana wahhabita.

${ }^{6}$ M. KHADDURI, al-Shâfi'î's Risâla. Treatise on the Foundations of Islamic Jurisprudence ", Johns Hopkins Press, Cambridge, 1997, pp. 35-36. 


\begin{abstract}
"Entre les grands imams qui travaillèrent consciencieusement à résoudre des questions légales et à former des jugements sur des points de droit, il existait une différence remarquable en ce qui regarde le nombre des traditions qu'ils ont fournies: les uns en possédaient une provision très grande, et les autres n'en avaient qu'une petite. On dit que le nombre des traditions enseignées par Abou Haniffa montait à environ dix-sept [ou à cinquante selon un autre rapport]. Le nombre de traditions saines possédées par Malek, et qu'il consigna dans le Mowatta, est d'environ trois cents. Ahmed Ibn Hanbel inséra trente ${ }^{7}$ mille des siennes dans son mosned. Chacun d'eux en a donné autant qu'il en avait pu recueillir par un travail zélé et consciencieux"8.
\end{abstract}

Le basi del poderoso edificio della legge islamica erano così state gettate e gli insegnamenti delle quattro scuole canoniche avevano raggiunto la loro maturità già nel $X$ secolo, tanto che si poté pretendere che fosse ormai chiusa la "porta dell'ijtihâd", cioè del lavoro diretto sulle fonti, e i secoli successivi hanno visto soprattutto rimaneggiamenti e commentari delle opere degli antichi capiscuola, con un conseguente distacco del diritto islamico dalle sue fonti originarie e dalla dinamicità che aveva fatto delle prime scuole dei veri laboratori di elaborazione del pensiero giuridico.

È così andata perduta la consapevolezza del loro ruolo e del processo che aveva condotto alla formulazione della Sharî' $a$ che spesso ancor oggi è vista quasi come qualcosa di disceso dal cielo similmente alla rivelazione coranica, piuttosto che un prodotto della storia, oppure come una sorta di codificazione di regole, cosa che non è mai stata presentandosi invece come un tipo di giurisprudenza basata su fonti eteroclite e sempre attenta alle circostanze spazio-temporali in cui andava esprimendosi. Ciò influisce ovviamente sul dibattito attualmente in corso riguardo a una delle principali rivendicazioni dei movimenti islamici radicali: il ritorno all'applicazione integrale della Sharî'a, in gran parte sostituita o affiancata da codificazioni di stampo moderno nella maggior parte dei paesi musulmani da un paio di secoli a questa parte.

Al di là dei suoi aspetti tecnici, la discussione in atto riflette varie posizioni che coinvolgono una questione di capitale importanza: col mutare delle condizioni sociali e culturali quale parte della tradizione islamica e delle istituzioni musulmane classiche vanno considerate ancor

\footnotetext{
7 Variantes: quarante, cinquante.

8 IBN KHALDOUN, Les Prolégomènes, traduits en français et commentés par M. DE SLANE, deuxième partie, Imprimerie Impériale, Paris, 1865, p. 360.
} 
valide e quindi mantenute? Quali aspetti sono invece modificabili e attraverso quali procedure? Per rispondere a queste domande è necessaria una riconsiderazione del processo evolutivo che nei primi secoli della storia dell'Islam ha condotto alla formazione delle sue dottrine e delle sue strutture fondamentali per poter riprendere, in forme adatte ai nostri tempi, il fecondo lavoro di quelle prime generazioni di credenti. Come vedremo, è questo uno dei temi oggi maggiormente dibattuti da musulmani di diverse tendenze che propongono a riguardo valutazioni e soluzioni differenti a talora addirittura opposte.

\section{5 - Rottura di un equilibrio}

Tutto sommato le norme del culto, ma anche molte disposizioni per numerosi casi concreti della vita individuale e collettiva, restarono in vigore a lungo in società sostanzialmente tradizionaliste e dove anzi le vicissitudini politiche avevano interrotto dinamiche fortemente evolutive proprie dei primi secoli, per lasciar spazio al lungo periodo della cosiddetta decadenza.

Fu proprio allora che si vide per così dire ipostatizzare il concetto $\mathrm{e}$ la funzione della sharî́a: il caos seguito alle devastazioni operate dai Mongoli, la fine delle grandi dinastie, la parcellizzazione dell'impero islamico partite dal XIII secolo, con i concomitanti attacchi delle crociate e la Reconquista in Spagna, oltre ai mai sopiti conflitti settari interni alla Umma, fecero percepire il campo del diritto come l'ultimo baluardo per difendere l'Islam da un possibile collasso totale 9 .

È stato però solo dal XVIII secolo in poi che il sistema si è trovato a essere superato dai fatti e non poche sono state le responsabilità delle potenze coloniali - la Gran Bretagna in India, l'Olanda in Indonesia, la Francia altrove, e insieme a Gran Bretagna e Russia nei confronti dell'impero Ottomano - nel favorire o imporre innovazioni che erano

9 Dal punto di vista più tecnico del diritto la questione andrebbe articolata più dettagliatamente. Ci limitiamo a rimandare agli studi specialistici in materia quali: $\mathbf{R}$. ALUFFI BECK PECCOZ, Il modello giuridico, scientifico e legislativo, italiano in Tunisia e Marocco, in S. LANNI e P. SIRENA (a cura di), Il modello giuridico-scientifico e legislativo italiano fuori dell'Europa, Atti del II Congresso Nazionale della SIRD (Siena, 2012), Edizioni Scientifiche Italiana, Napoli, 2013, pp. 37-46; F. CASTRO, La codificazione del Diritto privato negli Stati arabi contemporanei, in Rivista di Diritto Civile, n. 4 (1985), pp. 387-447; J. SCHACHT, Introduzione al diritto musulmano, Fondazione Giovanni Agnelli, Torino, 1995; WAEL B. HALLAQ, Introduzione al diritto islamico, il Mulino, Bologna, 2013. 
finalizzate a tutelare i propri interessi o a sistematizzare ciò che ai loro occhi appariva incongruo o caotico, ponendo così le basi di una crisi profonda e irreversibile nella visione classica del diritto islamico.

Non solo nuovi istituti e norme di stampo occidentale si affiancarono a quelle tradizionali, ma la stessa idea di codificare ciò che per secoli non lo era mai stato e la trasformazione delle istituzioni locali sul modello degli stati moderni europei ebbero alla fine un effetto dirompente.

Se le leggi del cosiddetto statuto personale rimasero più a lungo 'protette' da troppo vistosi mutamenti, ciò dipese sia dalla volontà dei musulmani di continuare ad aderirvi, sia dallo scarso interesse delle potenze coloniali a mettervi mano. Ma ciò, in definitiva, portò a una specie di sdoppiamento del sistema giuridico in cui coesistevano due filoni paralleli e indipendenti che alla fine non avrebbero potuto perdurare senza gravi squilibri e contraddizioni.

Le classi dirigenti locali occidentalizzate e nazionaliste che già nel periodo coloniale avevano accettato se non favorito tale processo, anche dopo l'indipendenza proseguirono sulla stessa rotta per rafforzare stati burocratici e fortemente centralizzati e timorose che le differenze etniche e religiose presenti sul terreno potessero favorire spinte autonomiste se non separatiste.

In campo giuridico, come pure in altri settori del pensiero islamico moderno e contemporaneo, si delinearono presto due opposte attitudini di fronte alle sfide poste dalla modernizzazione in corso nei paesi musulmani: da un lato alcuni si resero conto dell'inevitabilità di profonde trasformazioni anche interne al sistema e non esitarono nel promuoverle, distinguendo ciò che andava preservato soprattutto dello spirito della propria tradizione e quel che si doveva invece modificare nelle forme; dall'altro sorse presto un movimento di rifiuto della modernizzazione (ritenuta un cedimento verso modelli alieni e perniciosi) che tendeva invece a preservare alla lettera disposizioni e a mantenere se non a ripristinare a ogni costo qualsiasi cosa fosse percepita come parte dell'eredità dei padri, oltretutto immaginati quali rappresentanti quasi di un mito perduto: la civiltà classica dell'Islam trionfante proprio in quanto obbediente ai dettami divini.

Il momento storico favorì per un certo periodo i primi, ma le forti tensioni persistenti e gli scarsi successi ottenuti dopo la decolonizzazione, portarono gradualmente i secondi a prevalere, con un'opposizione non soltanto verso le potenze straniere, ma anche verso i regimi locali che andavano sostituendo la tutela di quelle, ma per vari versi ne proseguivano le politiche occidentalizzanti. 
La retorica di una necessaria 'riforma' si imponeva a 360 gradi, ma mentre per qualcuno si trattava di un autentico 'rinnovamento' (tajdìd) per molti altri, impregnati di uno spirito di rivincita, si preferiva una sorta di 'restaurazione' (islàh), tratto questo comune a molti 'modernismi' religiosi che alla fine si limitano a un atteggiamento apologetico secondo il quale già tutto è compreso e risolto nel modello originario (né potrebbe essere diversamente, considerandolo di origine divina e avulso da qualsiasi contestualizzazione storica che ne riveli anche le dinamiche evolutive interne e l'apporto umano che vi contribuì) il quale andrebbe quindi semplicemente 'ripristinato'.

Gli slogan nazionalisti e panarabisti entrarono definitivamente in crisi con la bruciante sconfitta nella Guerra dei Sei Giorni (1967) e il cosiddetto 'islam politico' ne trasse vantaggio rispetto ad altre opposizioni di tipo laicizzante, del resto eliminate o ridotte al lumicino dai regimi in carica tramite una politica illiberale che seppe sfruttare ai suoi fini egemonici anche gruppi musulmani radicali che insipientemente li coadiuvarono non poco a tal fine.

Non a caso una delle rivendicazioni principali di questi ultimi fu proprio il ritorno all'applicazione della sharî́a, sotto il celebre slogan banalizzante 'l'islam è la soluzione' duramente contestata da recenti studiosi indipendenti ${ }^{10}$.

\section{6 - Emanciparsi dalla sharî́a o applicarla? La disputa fra Corano e Sunna}

Come abbiamo già accennato, la gran parte delle disposizioni sciaraitiche non avrebbero potuto essere elaborate senza l'apporto fondamentale e dirimente della Sunna. I relativamente scarsi e talvolta generici versetti prettamente normativi del Corano, persino in materia di culto, hanno trovato nei detti del Profeta il completamento necessario divenendo progressivamente ancor più cogenti del Testo 'rivelato' in materia precettistica.

Va però anche tenuto conto che fra le migliaia di detti attribuiti al Profeta ve ne sono d'ogni tipo: narrativi, esortativi, edificanti, legati a usi e abitudini dell'Arabia antica o dei territori in cui l'Islam si stava espandendo, finendo per affastellare un'enorme quantità di materiale eterogeneo quanto ad argomento e rilevanza, talvolta con tutta evidenza

${ }^{10}$ W.B. HALLAQ, Introduzione al diritto islamico, il Mulino, Bologna, 2013. 
finalizzato a sostenere o perorare la causa di gruppi tribali, dinastie, etnie e fazioni in cui era divisa la comunità musulmana fin dal suo esordio.

Il lavoro di verifica dei pur scrupolosi redattori delle principali 'raccolte autentiche', basato essenzialmente sulla verifica della talvolta lunghe catene dei trasmettitori piuttosto che sulla consistenza e coerenza del passo riportato, lasciò inevitabilmente convivere 'detti' anche contraddittori (tra loro o con lo stesso Corano), relativi ad ambiti di vario spessore, dal diritto penale a norme di galateo, fino ad alcuni persino per certi aspetti bizzarri (esempio, 'L'amore per i gatti fa parte della fede').

L'impegno degli estensori di raccolte 'genuine' era stato motivato da esigenze di credibilità e autorità di un corpus che non poteva accogliere indiscriminatamente ogni cosa anche e soprattutto per non compromettere il proprio ruolo di fonte attendibile di riferimento.

È evidente che l'attuale rivendicazione di molti movimenti islamisti per una reintroduzione integrale della sharî́a altro non è che un modo, forse l'unico a disposizione, per opporsi a regimi autoritari e corrotti, apparentemente inamovibili salvo il caso della Tunisia post Primavere arabe, altrove degenerato in forme di autocrazia ancor peggiori quando non in guerre civili devastanti.

'Basterebbe seguire i dettami divini' parrebbe una posizione comprensibile, ma il trucco sta nel ritenere questi ultimi qualcosa di ben definito e inalterabile, codificabile e applicabile senza mediazioni che invece sono state le costanti del diritto islamico, ma evidentemente nessuno slogan può tener conto delle articolazioni della storia, poiché perderebbe ogni efficacia dovuta appunto alla semplificazione e banalizzazione di quanto reclama, necessariamente senza alcuna consapevolezza delle molte problematiche che esso comporterebbe se fosse seriamente affrontato.

Hussein Ahmad Amin (1932-2014) è stato tra i più significativi autori egiziani che potremmo definire 'figli della Nahda', ossia del vasto movimento di risveglio culturale arabo fiorito tra 1800 e 1900. Nel suo caso non si può usare questa espressione soltanto in forma figurativa, in quanto egli è nato proprio da uno dei 'padri' della stessa Nahda, universalmente noto per le sue opere anche fuori dal mondo arabo: Ahmad Amin (18861954), studente e sodale del celebre rettore riformista di al-Azhar Muhammad 'Abduh, in seguito giudice e infine Preside della Facoltà di Lettere dell'Università del Cairo. Tra le sue numerose opere quella destinata al maggior successo fu una storia della cultura islamica concepita insieme a Taha Husayn e composta da vari volumi scritti tra il 
1933 e il $1952^{11}$. Rispetto a successivi testi di carattere più apologetico o polemico, nati nel periodo della lotta per l'indipendenza e la decolonizzazione, questi possono essere riconosciuti come il frutto intellettualmente più maturo della rinascita culturale dell'Islam contemporaneo. Come farà anche il figlio, l'autore tentò anche un approccio storico-critico della Sunna, pur rimanendo nel solco degli studi tradizionali e senza approdare a quella sorta di demitizzazione che invece osò in seguito Hussein, come vedremo, comunque debitore rispetto al genitore di una formazione di altissimo livello ricordata con affetto e nostalgia nella propria autobiografia Fī bayt Ahmad Aminn (A casa di Ahmad Amin) ${ }^{12}$. Hussein ebbe una formazione essenzialmente giuridica, completata da studi letterari in Inghilterra, per poi lavorare all'Arabic World service della BBC e infine entrare nella diplomazia egiziana divenendo anche ambasciatore. Dalīl al-Muslim al-haziñ ilā muqtada-l-sulūk fíl-garn al-'ishrin (Una guida per il povero e perplesso musulmano al tornante del XX secolo) per insieme al suo altro libro Hawla al-da 'wa 'ilā tatbīq al-sharī a (Circa l'appello all'applicazione della Shari'a) ${ }^{13}$ produssero vaste polemiche anche al di fuori dell'Egitto. Non certo paragonabile alla celebre Guida dei Perplessi di Maimonide (1135-1204) ne riecheggia tuttavia il titolo e soprattutto lo spirito ben rappresentato da questa massima del grande pensatore ebreo del medioevo: "Non considerare una cosa provata soltanto perché la trovi scritta in un libro, chi sa mentire con la lingua non avrà remore a farlo anche con la penna".

Pur coronato dal Premio per la miglior opera dell'anno alla Fiera del libro del Cairo nel 1984, il libro suscitò aspre critiche non solo In Kuwait, Qatar, Abu Dhabi e Bahrein, ma sullo stesso quotidiano cairota alAhram, benché altri, come i noti giornalisti Anis Mansur sulla rivista October e Fathi Radwan su al-Hilal ne tessero le lodi. Le polemiche indussero l'autore ad approfondire e ad ampliare le tematiche trattate che confluirono nelle successive edizioni del volume che affronta con audacia argomenti quali le moderne biografie del Profeta, l'autenticità dei Detti a lui attribuiti, le ragioni politiche delle divisioni fra musulmani di diversa

11 A. AMIN, Fajr al-Islām (L'alba dell'Islam, 1 volume), Cairo, 1938; Duhāa al-Islām (L'aurora dell'Islam, 3 volumi), Cairo, 1938; Zuhr al-Islām (Il meriggio dell'Islam, 4 volumi), Cairo, 1952, in seguito condensati in un unico testo: Yawm al-Islām (I giorni dell'islam), sempre per i tipi di Lajnat al-ta'lif, wal-ltarjama, wa-l-nashr, Cairo, 1952.

12 H.A. AMIN, Fi bayt Ahmad Amīn (Nella casa di Ahmad Amin), Dar al-Hilal, Cairo, 1989.

13 H.A. AMIN, Hawla tatbiq al-shari'a al-islamiyya (Circa l'applicazione della sharî'a), Madbuli, Il Cairo, 1987. 
tendenza (ad esempio, Sunniti e Sciiti), il sufismo ... basandosi sulle opere della grande stagione dell'umanesimo musulmano che raggiunse il suo apice nella Baghdad del periodo abbaside.

I meriti delle sue sane provocazioni sono stati riconosciuti dal Marocco all'Indonesia, dove molti hanno apprezzato le due intenzioni dell'autore, apparentemente contraddittorie ma in realtà complementari: rimanere fedele alla propria origine e identità religiosa ed emendarla dalle attitudini che rischiano di porla in conflitto con i mutamenti dell'era moderna. Tuttavia il clima dominante in molti paesi arabi circa tali questioni è ben evidenziato dalle condanne subite da alcuni che hanno osato troppo: il caso più grave è accaduto proprio in Egitto quando, nel 1995, il professor Nasr Hamid Abu Zayd è stato accusato di apostasia a causa dei suoi studi innovativi sull'ermeneutica coranica e ha dovuto abbandonare il paese con la moglie, altri esempi più recenti sono: nel 2015 a Islam El-Behairy fu inflitto un anno di carcere per aver criticato alBukhari e Ibn Taymiyyah, nel 2016 a Fatima Naoot ne furono comminati 3 per le sue riserve sul sacrificio rituale di animali alla fine del Pellegrinaggio e nel 2017 al predicatore Mohamed Abdullah Nasr ben 5 per analoghi motivi. Per dare un'idea dello spirito critico dell'Autore vogliamo citare un passo di una sua opera:

\footnotetext{
"Benché anch'io mi auguri che la shari'a torni a essere applicata, non penso che ciò sia facile quanto sembrano credere quanti lo reclamano. Non si tratta infatti semplicemente di rimettere in vigore raccolte $\mathrm{o}$ codici di disposizioni legislative islamiche definitive, dettagliate e precise da parte di governi che le avrebbero a lungo ignorate. Opere di questo genere infatti non ne esistono. Tutto ciò che abbiamo, oltre al Corano e alla Sunna, non è che un ammasso di libri di giurisprudenza composti dagli esperti delle quattro scuole giuridiche... Molte delle disposizioni raccolte in queste opere sono contraddittorie e inconciliabili, e non sono mai state fatte oggetto di un'opera di armonizzazione né di una selezione che desse loro una forma definitiva, alla quale si sia convenuto di attenersi" [...]. Supporrei che la shari'a non dovrebbe essere passibile di modificarsi sulla scorta delle trasformazioni sociali, politiche ed economiche. Direi anzi che essa è l'insieme dei precetti che Iddio stesso ha stabilito per guidare ogni musulmano nel suo comportamento e nei rapporti col proprio Creatore e con gli altri uomini per quanto concerne il culto, la famiglia, l'azione sociale e politica. Eppure mi trovo davanti a enormi opere di giurisprudenza riguardanti precetti della shari'a talmente esaustivi da stabilire quali strumenti musicali siano leciti e quali no; il modo di accoppiarsi; come svolgere gare di corsa o altre competizioni; la raffigurazione di esseri viventi; la maniera di vestirsi
} 
e di acconciarsi; il risarcimento da versare per l'uccisione di un cane; l'allattamento di un adulto; il ripudio dovuto a cattiva conoscenza della lingua araba; la responsabilità di chi abbia acceso un fuoco per cucinare e, addormentatosi, abbia provocato un incendio di cose appartenenti al altri; di chi abbia spezzato un osso a un morto; di chi calunni un altro sostenendo che è figlio di suo zio o del secondo marito della madre o di uno straniero; il furto di vino o di un maiale di proprietà di un musulmano o di altri e così via. [...] Questi e altri simili precetti fanno veramente parte della shari'a? Sono vincolanti? E se sì, qual'è il loro fondamento divino? Il numero delle disposizioni legali prescritte esplicitamente nel Corano e nelle tradizioni universalmente ritenute validi è estremamente scarso se paragonato a quelle contenute nei libri di giurisprudenza. Nel Corano infatti non ci sono che un'ottantina di versetti d'argomento giuridico, come quelli relativi alle pene previste per il furto e per l'adulterio o concernenti il testamento e l'eredità. La maggior parte di questi versetti inoltre, si limita a enunciare principi generali che consentono interpretazioni e applicazioni differenti, passibili di essere adattati alle esigenze delle varie epoche e alle diverse circostanze. La Sunna autentica a sua volta si è limitata, sulla base dell'esempio e degli ordini del Profeta, a stabilire alcune norme relative alla guerra, alla politica e al culto (come il modo di compiere la preghiera) e ad introdurre delle rettifiche su base religiosa alle consuetudini relative allo statuto personale in vigore prima dell'Islam, conformemente alle mutate circostanze. [...] Non è quindi corretto pretendere che il Corano e la Sunna abbiano stabilito norme dettagliate e precise su tutti gli aspetti della vita dei musulmani: è più esatto dire che, durante la vita del Profeta, in molti campi si continuò ad attenersi al diritto consuetudinario dell'epoca preislamica. [...] Le relazioni tra la shari'a e il diritto consuetudinario non solo si perpetuarono ma si svilupparono per influsso delle ampie conquiste, infatti i popoli dei territori conquistati influenzarono la shari'a con i costumi invalsi presso le loro più complesse civiltà adducendo la motivazione che le semplici norme e i principii generali portati dagli arabi non erano sufficienti per organizzare i loro affari o che era difficile applicarli in condizioni differenti. L'innesto della sharîa sulle consuetudini di questi popoli facilitò l'accettazione dell'Islam da parte loro, ma ne derivò pure una diversificazione dei precetti della shari'a da paese a paese [...]. I popoli però trovano spesso mezzi efficaci per aggirare gli ostacoli e le rigide disposizioni che governanti e giuristi pongono sul loro cammino. I musulmani quindi, per aggirare l'ostacolo di questa seconda fonte del diritto, inventarono hadith nei quali inserirono quanto essi ritenevano adatto al progresso, attribuendoli poi al Profeta mediante una catena di trasmettitori creata a bella posta, redigendoli in uno stile vicino a quello del suo tempo e 
considerandoli poi validi e vincolanti per ogni epoca e per ogni luogo. Quando appariva un'altra generazione, con nuove esigenze, produceva altre 'tradizioni' nelle quali tali esigenze, contrastanti con quelle delle generazioni precedenti, trovavano espressione e anche questi venivano attribuiti al Profeta e ritenuti validi e vincolanti universalmente. Un altro mezzo efficace a cui si ricorse fu quello del consenso: cioè l'insieme delle dottrine e delle norme sulle quali, in una determinata epoca, vi era l'accordo tra gli esperti [...]. I sostenitori di questo principio si basano sul detto in cui il Profeta afferma: "La mia Comunità non concorderà mai su un errore". Molti non ritengono autentico questo detto, tanto più che la storia ha ben dimostrato che la Comunità musulmana non riesce mai a mettersi d'accordo su niente, si tratti o no di un errore [...]. Visto che stiamo parlando della campagna finalizzata all'applicazione della shari'a nella società contemporanea, devo far notare che, attenendosi al principio del consenso, è avvenuto che quanto era considerato un'innovazione riprovevole in una certa epoca è divenuto un obbligo vincolante per i musulmani del periodo successivo, nonostante la precedente condanna. Così chi invita a reintrodurre la consuetudine dei primi credenti tornando allo stato in cui erano le cose al tempo del Profeta, dei suoi Compagni e seguaci finisce per essere un eretico! Un esempio è la festa della nascita del Profeta. Fino all'ottavo secolo dell'Egira (il quattordicesimo dell'era cristiana) gli ulema hanno sostenuto che essa era contraria alla Sunna e la maggior parte di loro la proibì considerandola un'innovazione riprovevole. Eppure da quel tempo divenne una consuetudine profondamente radicata nella vita dei credenti e se oggi qualcuno vi si opponesse sarebbe preso di mira dagli uomini di religione che lo considererebbero un miscredente. Così pure in passato le preghiere rivolte ai santi o l'affermazione dell'impeccabilità del Profeta, rifiutate un tempo, tramite il consenso sono oggi state accettate e hanno sostituito le consuetudini dei primi credenti. Se teniamo conto che la maggior parte delle norme della shari'a ricevono il loro carattere imperativo dal principio del consenso, e che vi sono parti estremamente importanti della shari'a come quella sul Califfato - che non hanno altro fondamento che il consenso, come possiamo pretendere un'obbedienza totale a norme che variano nel tempo, stabilite da uomini dotati della nostra stessa capacità di riflettere, norme che una generazione ha considerato valide, che la generazione seguente ha rifiutato ritenendole eretiche e che una terza generazione è tornata a rispettare? Come possiamo dire che tali norme sono valide per ogni tempo e luogo e che i governi vi si devono attenere pena l'accusa di miscredenza e la minaccia di rovesciamento? Il fatto di trascurare le considerazioni storiche e il principio di evoluzione, l'incapacità di comprenderli e di farne uso è stato uno dei difetti del pensiero islamico e dell'immagine che $i$ 
musulmani si son fatti della loro religione. Chi studia la storia dell'Islam comprende che la shari'a è un palazzo elevato i cui piani sono stati costruiti uno dopo l'altro nel corso di lunghi secoli, da uomini simili a noi, in funzione dell'evoluzione della società e delle sue esigenze [...]. Se i musulmani desiderano veramente affrontare le sfide del tempo presente, penso che sia ora che abbandonino questa concezione statica e semplicistica delle cose e che comprendano la realtà delle fasi attraverso le quali la shari'a è stata edificata. Se non lo faranno, con tutta probabilità passeranno ancora lungo tempo a occuparsi di come portare la Jabaliya, se sopra o sotto le caviglie, della necessità di mangiare e bere usando la mano destra, della liceità dell'acquisto di fotografie, o chiedendosi se bere stando in piedi sia contrario alla Sunna, se convenga portare il bastone come espressione del proprio attaccamento all'Islam, di quali problemi comporti pregare vicino a una donna o le conseguenze in diritto di famiglia derivanti dal matrimonio con un jinn in forma umana o se infine mangiare seduti attorno a una tavola sia dimostrazione di scarso attaccamento alla Sunna e al Profeta. E tutto ciò mentre le altre nazioni si occupano di cose ben differenti e preferiscono agire che discutere" ${ }^{\prime 14}$.

Anche se non si tratta di un giurista, le sue osservazioni conservano la loro validità, come a volte succede - e lo stesso linguaggio lo registra quando si dice 'pur essendo un profano' (cioè non addetto al 'tempio') non intende certo affermare che siano cose insensate. Anzi, chi si esprime anche e soprattutto in nome di 'altro', non credo abbia il diritto né l'interesse a parlare come se non vivesse su questo pianeta, pena la sua stessa credibilità. Non è certo unicamente l'incarnazione cristiana a doversi misurare con le coordinate spazio-temporali della storia. Lo stesso cervello umano che si è evoluto in milioni di anni e comunque si sviluppa fino a una certa maturità molto più lentamente rispetto ad altri animali lo stanno a dimostrare.

\section{7 - Solo il Corano basta ${ }^{15}$}

Uno dei primi esponenti di questa tendenza fu già nel periodo abbaside (750-1258) Ibrâhîm al-Nazzâm (m. 835) teologo e giurista che rifiutava

\footnotetext{
${ }^{14}$ H.A. AMIN, Hawla tatbiq, cit., p. 186 ss.

15 Cfr. A. DUDERIJA, The Sunna and its Status in Islamic Law: The Search for a Sound Hadith, Palgrave Macmillan, London, 2015.
} 
l'autorità dei hadith, facendo riferimento al solo Corano. Come altri mu'taziliti, prima vera e propria scuola di teologia musulmana ai tempi del califfo al-Ma'mūn, egli diffidava specie delle tradizioni riportate da Abū Hurayra, il narratore forse più citato nella Sunna.

Come si è detto, tuttavia, le conseguenze sul piano religioso non intaccano la dottrina, bensì alcuni dettagli nei rituali: dalla professione di fede limitata alla prima delle due affermazioni (quella che ricorre appunto nel Corano: la ilâha illâ Allâh 'non c'è altro dio che Allah') tralasciando wa Muhammad rasūl Allâh (e Maometto è il Suo inviato); il periodo in cui ritengono valido il pellegrinaggio, che può essere allungato a tre mesi e non limitato a uno solo, e il rifiuto di baciare la Pietra Nera; anche se pregano 5 volte al giorno ritengono che le orazioni quotidiane prescritte dal Corano siano solo 3; ammettono al culto le donne mestruate e le accettano anche come guide della preghiera comunitaria e come predicatrici; versano l'elemosina legale più spesso che una sola volta all'anno; non praticano la circoncisione; ammettono il consumo moderato di alcolici purché non alterino il comportamento dell'orante; non ritengono i cani animali impuri; non proibiscono musica e danza e non impongono determinati abiti, neppure il velo alle donne.

Nei primi secoli dell'Islam la questione più delicata e dibattuta a proposito del Corano, analogamente a quanto era avvenuto tra i primi cristiani sulla natura umana e divina del Cristo, fu quella sul suo carattere di Verbo 'creato' o 'increato' di Dio. Col tempo si affermò la seconda opzione, e non è il caso di approfondirla in questa sede, ma non si può evitare di sottolineare il paradosso di un Testo eterno e increato dove tuttavia appaiono sia risposte a situazioni ben concrete prodottesi durante la predicazione del Profeta ("Ti chiederanno ... rispondi ..." con un'intera letteratura sulle circostanze, motivazioni o cause della rivelazione dette in arabo Asbâb a-Nuzūl), sia disposizioni che ne modificano o ne abrogano altre date in precedenza.

Il problema della relazione trascendente/immanente o assoluto/storia si pone in questo caso come articolato e complesso. Ma per i semplici, paragonabili ai nostri lettori dei Fioretti di s. Francesco o ai più eruditi che si appassionavano alla Imitazione di Cristo la filologia non era certo la questione di maggior rilevanza, con l'aggravante che nell'Islam il solo Profeta è degno di venerazione, per cui ogni cosa riferita alla sua persona e ai suoi atti viene sempre tenuta in massima considerazione. Dispute dottrinali non erano assenti, ma riguardavano ristrette élites, mentre quelle dinastiche si trascinavano senza sbocco, anche per queste motivazioni il dibattito sulla Sunna, la sua autenticità e il suo valore normativo ebbe una risonanza maggiore e continua a suscitare 
controversie, soprattutto dopo i rapidi e profondi cambiamenti che l'epoca contemporanea continua a produrre in ogni parte del mondo.

Non è forse un caso che tra i più recenti esponenti della tendenza per così dire 'coranista' figurino intellettuali musulmani del subcontinente indiano nell'epoca del dominio coloniale inglese o autori che hanno lavorato principalmente in Occidente. Per i primi fu probabilmente determinante il contesto di un paese mai completamente islamizzato e nel quale i musulmani rimanevano una forte minoranza che doveva misurarsi anche con altri gruppi etnici e religiosi in un ambito pluralistico, per i secondi il confronto con la modernità in atenei occidentali svolse un ruolo simile.

Tra i pionieri va citato almeno l'appunto indiano Ghulam Ahmad Parvez (d. 1985) che non esitò a rilevare come alcuni hadith stabiliscono pene quali la morte per apostasia o la lapidazione per adulterio, assenti invece nel Corano. Insomma, molte disposizioni oltre che esser dubbie e in contraddizione tra esse e col dettato coranico, sono probabilmente usi invalsi tra gli arabi nei due secoli successivi alla scomparsa del Profeta e non si vede la ragione di considerarli vincolanti e definitivi.

Il carattere innovativo del Corano sarebbe quindi stato depotenziato se non vanificato in tal modo, come ritiene un altro intellettuale d'origine pakistana docente a Chicago: Fazlur Rahman (d. 1988). Se il desiderio di uniformarsi alla condotta di Maometto non è di per sé criticabile, l'enorme espansione del credo islamico susseguita ha generato la Sunna che però fissa, non senza incertezze e incoerenze, una sola fase della successiva e lunga vicenda islamica nel mondo intero, pretendendo un carattere totalizzante nei minimi dettagli che mal si adatta a una tanto variegata situazione, una sorta di "umbrella of behavioural norms and an interpretive process by which Muslims could adapt their law to changing circumstances" 16 . Ne viene dunque contestata la rigidità.

Ciò non significa che anche arabi musulmani abbiano aderito alla corrente coranista.

Già a cavallo fra '800 e '900 il medico egiziano Muhammad Tawfiq Sidqi (d. 1920) riteneva poco attendibili oppure limitate al loro tempo molte disposizioni contenute nella Sunna.

Sempre egiziano fu Ahmed Subhi Mansour (n. 1949), che ha patito espulsioni e persino il carcere nel suo Paese d'origine, per trovare asilo

16 B. KRAWIETZ and H. REIFELD (Hrsg.), Islam and the The Rule of Law. Between Sharia and Secularization, Konrad-Adenauer-Stiftung e.V., Sankt Augustin, Berlin, 2008, p. 85. 
negli Usa dove si impegna per $\mathrm{i}$ diritti umani. In un'intervista ha dichiarato:

"If you look at many extremists you will find that they rely on the hadith and not the Quran," argues Mohamed, who remains keen, nonetheless, to stress that Quranists do not reject the hadith and Sunna out of hand. "We differentiate between issues like how to pray, fast and perform the Haj, practices passed down from generation to generation, and issues that are open to interpretation"17.

Forse più noto per la sua parentela con Hassan al-Banna, fondatore dei Fratelli Musulmani in Egitto nel 1928, è stato Gamâl al-Bannât8:

«Sostenitore di un Islam liberale, Gamâl al-Bannâ porta agli estremi la critica alle scienze religiose tradizionali che è una delle costanti del pensiero islamico contemporaneo. L'autore scava in tal modo un fossato tra la Parola di Dio, il Corano, e la parola degli uomini, compresa quella del Profeta dell'Islam. La mossa è duplice: consegnare la tradizione profetica al vaglio critico della storia e, per contrasto, accentuare ulteriormente la centralità del Corano. L'operazione è condotta con grande maestria e i vantaggi sono evidenti: sono de-sacralizzati e dunque relativizzati una serie di hadîth che oggi creano difficoltà ai musulmani nell'organizzare la società ("obbedisci al principe anche se ti picchia e ti ruba il denaro") o nel rapportarsi ai credenti delle altre religioni ("chi cambia religione, uccidetelo"). Al-Bannâ conclude in effetti la sua serrata argomentazione con un pezzo magistrale sulla giornata tipica di un musulmano passatista, inconsapevole vittima dei crimini della tribù del "Ci è stato riferito" (la formula con cui si apre ogni tradizione islamica). La condanna è senza appello: "Frutto della superstizione, questi hadith hanno annientato le anime e resa idiota e non creativa la mentalità dei musulmani“ (p. 183). [...] $\mathrm{Ne}$ risulta un'assolutizzazione del Libro, la cui logica conclusione è il rifiuto della dottrina dell'abrogante e dell'abrogato, secondo la quale il versetto coranico più tardo, solitamente più restrittivo, annullerebbe i precedenti, non di rado più universali. Questa duplice mossa - rifiuto degli hadîth e lettura globale del Corano - permette a Gamâl al-Bannâ di sostenere una visione moderna dell'Islam, non più vincolata ai modelli medievali. Ci si può tuttavia domandare se il ritorno alla

\footnotetext{
17 https://web.archive.org/web/20090910220251/http://weekly.ahram.org.eg/2007/852/eg12. htm

${ }_{18}$ Cfr. L'Islam, la liberté, la laïcité et le Crime de la tribu des "Il nous a été rapporté», a cura di G. Al-BANNA, D. AvON e A. EliAs con la collaborazione di A. IDRISSI, L'Harmattan, Paris, 2013.
} 


\begin{abstract}
pura lettera sia realmente possibile o se piuttosto la trascendibilità della storia resti una pericolosa illusione. Ė infatti da osservare che il tema del puro Corano è molto presente anche nella letteratura di tipo fondamentalista, laddove esponenti del sapere tradizionale, in particolare mistico, appaiono più consci della necessaria mediazione tra credente e Testo» 19 .
\end{abstract}

Per quanto esistente e assai poco noto, questo dibattito pur non trascurabile resta poco conosciuto fuori dal mondo islamico e anche al suo interno non ha schiere di seguaci. Del resto, i poco meno di due miliardi di musulmani attualmente al mondo sono per la maggior parte non arabofoni e anche tra gli arabi, a parte una consistente fetta di analfabeti, soltanto coloro che hanno fatto studi umanistici superiori sarebbero in grado di comprendere le sottigliezze ermeneutiche ed esegetiche di una simile diatriba. Com'era doveroso segnalarla, è altrettanto inevitabile rilevarne lo scarso seguito senza soffermarci in questa sede più in profondità e nei dettagli.

\title{
8 - La critica degli orientalisti
}

Già con Gustav Weil (1808-1889) gran parte della raccolta di al-Bukhari venne messa in dubbio ${ }^{1}$ mentre per William Muir (1819-1905) le catene dei trasmettitori garantivano poco per un materiale tutt'al più utile a ricostruire una specie di biografia di Maometto, per la quale assolutamente insufficienti sarebbero stati i pochi passi coranici a disposizione, specialmente per la lunga fase di attesa (40 anni circa) prima della 'rivelazione' e della conseguente predicazione.

Ma fu l'ungherese Ignaz Goldziher (1850-1921) il primo a dedicarsi a una più sistematica e approfondita analisi della questione. La mancanza di documenti scritti risalenti a prima di un secolo dopo la morte del Profeta ne metteva in dubbio l'autenticità, così come alcune delle tematiche da essi trattate, inerenti a situazioni determinatesi solo dopo la sua scomparsa e fortemente legate a tensioni interne alla Umma. Inoltre, e paradossalmente, erano in maggior numero le testimonianze risalenti non tanto alla prima generazione dei suoi Compagni, quanto a quella successiva, che trasmise detti e aneddoti precedentemente del tutto assenti. Quelli relativi ai meriti o demeriti di questa o quella casata pretendente al ruolo di Califfo (gli Alidi o Sciiti da un lato, i Sunniti prima

19 https://www.oasiscenter.eu/it/critica-agli-hadith-gamal-al-banna 
Omayyadi, poi Abbasidi dall'altro) inducono a ipotizzare una vera e propria 'produzione' di materiali funzionali alla lotta politica in corso. In fin dei conti, non essendoci modo di stabilire l'autenticità del materiale raccolto nella Sunna, essa venne accettata in base a tale approccio critico non tanto in quanto testimonianza di ciò che il Profeta avrebbe detto o fatto realmente, ma come frutto delle tensioni interne e alle differenti posizioni emergenti nell'Islam delle origini. Quest'ultimo necessitava di una base di riferimento da accostare al Corano per istituzionalizzarsi e questa fu la funzione delle tradizioni attribuite al Profeta che aumentavano di numero e d'importanza, dotandosi di catene di trasmettitori più articolate e precise col passare del tempo, nell'arco grosso modo dei due prime secoli dell'era islamica.

Un ulteriore contributo allo studio del diritto islamico si deve a Joseph Schacht (1902-1969) che non si limitò allo studio della Sunna, ma la pose tra gli elementi chiave della prima evoluzione del sistema giurisprudenziale musulmano Concentrandosi sulla datazione degli hadith, notò che anche in delicate questioni 'teologiche' alcuni di essi, e di notevole rilevanza, non erano mai stati citati prima dell'epoca omayyade, proprio quando ricorrere a tali testi sarebbe stato utile se non risolutivo nelle dispute che si stavano sviluppando in quelle che potremmo definire le prime correnti del pensiero religioso islamico, non ancora vere e proprie scuole, ma concentrate su argomenti di capitale importanza sui due versanti dogmatico e giuridico (strettamente correlati), come ad esempio quello della predestinazione opposta al libero arbitrio, determinante nel definire lo status del musulmano 'peccatore', in primis ovviamente del sovrano e della sua cerchia che potevano o meno essere considerati legittimi nonostante più o meno gravi mancanze da parte loro nei confronti delle norme islamiche, favorendo un quietismo utile al mantenimento dello status quo nel primo caso, mentre nel secondo si sarebbero facilitate se non incoraggiate posizioni critiche se non di vera e propria sedizione e rivolta verso il potere costituito. La mancata menzione di hadith dirimenti o comunque di peso su determinate questioni fa propendere lo studioso a ritenere che non esistessero ancora. Del resto nella sua analisi di una delle prime raccolte, la celebre Muwatta' (93-179), meno di un terzo degli hadith sono riferiti direttamente al Profeta, mentre il resto risalgono a suoi Compagni o a successori di questi, mentre in seguito, e soprattutto dopo la canonizzazione della Sunna da parte di alShafi'i (150-204), la catena di trasmissione risale quasi sempre a Maometto, anche nel caso di hadith già noti in precedenza, ma la cui origine si fermava a uno dei suoi Compagni o addirittura a qualche loro successore. 
Per farsi una ragione di tali apparenti incongruenze vale la pena di ricordare la dinamica delle prime tappe dell'espansione musulmana nel primo secolo: dette 'razzie' (maghàzi o ghazawàt) per divenire conquiste (futùhàt) permanenti esse videro installarsi in nuovi e vasti territori delle guarnigioni stanziate nei pressi delle principali città, dove la maggioranza della popolazione non era ancora convertita all'Islam e pertanto seguiva i dettami della propria fede di appartenenza e le tradizioni locali. Queste ultime furono incorporate tra l'altro nel diritto islamico in formazione nella categoria 'urfi (appunto tradizionale) che ancora permane fino a oggi: per esempio vale a dire che purché rispetti alcuni requisiti come la presenza di testimoni e il versamento della dote, un matrimonio è valido anche se non registrato presso le istituzioni statali competenti.

Da ciò si può dedurre che i primi giuristi musulmani, in base alla loro conoscenza del Corano e del contesto in cui operavano, ispirandosi anche a usi e costumi dei gruppi tribali che costituivano il grosso della comunità musulmana protagonista delle conquiste, potevano sentenziare fondandosi sul proprio parere e furono detti pertanto ahl al-ra'y (quelli che ricorrono alla propria opinione), ben presto in contrasto con i tradizionisti detti pertanto ahl al-hadith (cioè coloro che si basavano su disposizioni riferite a insegnamenti o comportamenti dei primi seguaci del Profeta o di Maometto stesso).

Basti inoltre pensare che fra i territori conquistati per primi rientravano intere e antiche civiltà 'idrauliche' e agricole (come la Mesopotamia e l'Egitto) o zone che avevano a lungo conosciuto l'amministrazione persiana o bizantina ... i califfi e i loro collaboratori non avrebbero potuto neppure immaginare di poter governare simili terre basandosi sul solo Corano o sugli usi propri dei nomadi e senza l'ausilio di funzionari locali anche non musulmani che non a caso rimasero al loro posto, come del resto i medici di corte, gli astronomi ... fino ai ciambellani e ai musici, indispensabili per chi si riteneva erede di un potere imperiale e inoltre più fidati di conterranei o correligionari necessariamente influenzati da appartenenze (e ruggini) intertribali oltre che coinvolti a vario titolo nelle dispute di potere che ben presto travagliarono la neonata comunità. Dei primi quattro califfi pur denominati 'ben diretti' (ràshidùn) ben tre morirono di morte violenta ... La mitica e confortante denominazione perdura tutt'oggi, pur essendo universalmente nota la piega che presero gli eventi dopo i primi due (Abu Bakr e Omar, suoceri del Profeta) seguiti dall'altra coppia (suoi generi) che vide nascere la spaccatura fra sciiti e sunniti, né sembra inquietare più di molto che di tutta la storia del califfato (abolito nel 1924 dal fondatore della Repubblica 
di Turchia) si salverebbe così soltanto il primo trentennio, tra l'altro assai meno concorde e pacifico di quanto lo si voglia rappresentare.

\section{9 - Ulteriori studi: ICMA (isnad-cum-matn analysis)}

Questa nuova metodologia che si basa sull'analisi parallela del contenuto della tradizione e della sua catena di trasmettitori è stata intrapresa nel 1996 da Gregor Schoeler and Harald Motzki.

Prendendo in esame tutte le raccolte della Sunna e non solo le sei principali è stato loro possibile tramite lo studio degli hadith con isnad corti o interrotti, ipotizzare per alcuni delle precedenti versioni che ne costituirebbero la genuina o comunque meno dubbia origine.

Ne sono stati ricavati complessi diagrammi la cui finalità è stata chiaramente indicata da Motzki, uno degli autori:

"I only assume that many traditions found in the extant compilations were not invented by the compilers but have a history (including forgeries) which can be retraced to a certain point in time. The main aim of my approach is dating traditions. The fact that, for example, a hadith of the Prophet can be dated to the second half of the first/seventh century does not mean that it is authentic in the sense that it really goes back to him in the form preserved or that it reflects accurately what really happened" 20 .

Una più ampia ricognizione storica e su un più vasto corpus, dando peso alle varianti testuali del fatto riportato può certamente fornire elementi per una datazione più precisa degli hadith o l'individuazione di una radice comune fra quelli similari. Temiamo tuttavia che questo tipo di studi iper-specialistici abbiano poca probabilità di incidere sulla percezione del valore legale di tali documenti sia rispetto al grande pubblico, sia nei confronti della maggioranza dei giurisperiti che si trovano quotidianamente ad affrontare quesiti ai quali rispondono con le famose fatwa, basate in sostanza su un ricorso alla Sunna tradizionale e sempre più evidentemente decontestualizzato, come ha clamorosamente dimostrato l'esempio citato in apertura.

${ }^{20}$ H. MOTZKI, N. BOEKHOFF-VAN DER VOORT, S.W. ANTONY, Analysing Muslim Traditions. Studies in Legal, Exegetical and Maghāzì Hadìth, Brill, Leida, 2010, pp. 234-5. 


\section{0 - Maqàsid al-shari'a (le finalità della legge islamica)}

La questione, pur in modalità differenti, si è posta e si pone anche in altre religioni, specie in quelle cosiddette rivelate. Sia nella 'rivelazione' propriamente detta che può essere intesa come 'ispirata' o invece una vera e propria sorta di 'dettatura', sia per altre 'fonti' in qualche modo 'garantite' dal Rivelatore che si appalesa in tal modo anche come Legislatore.

La messa per iscritto della 'rivelazione e la codificazione della normativa da essa dedotta anche, e talvolta soprattutto, grazie ad altre 'fonti' ausiliarie ha conosciuto tempi, forme e problematiche differenti lungo il corso di secoli se non di millenni. La maturazione di tale processo è stata di volta in volta considerata matura se non definitiva in epoche differenti, sia comparativamente fra le diverse tradizioni religiose, sia all'interno di ciascuna di esse.

Eppure proprio uno dei detti di Maometto predice l'avvento, ogni cento anni, di un mujaddid o riformatore dell'Islam: il problema resta, non a caso, come interpretare tale 'riforma'. Lo stesso termine può indicare il ritorno a una forma primigenia e autentica, corrottasi col tempo (ma in questo caso il termine arabo è piuttosto islàh). Sarebbe dunque più esatto definirla una 'restaurazione'. Altra possibile ed effettiva concezione (presente nella radice stessa del termine mujaddid) intende invece un 'rinnovamento'. Se nell'Islam non si è praticamente mai posto il tema di un mutamento dei riti e delle liturgie, considerati 'diritti di Dio' e dunque non modificabili, per tutto il resto vi sono stati e permangono movimenti o correnti favorevoli ad autentiche innovazioni e a superare forme e interpretazioni ormai considerate improponibili.

Le pur sofisticate tecniche proposte per verificare l'autenticità dei detti profetici, rivolte a un ristretto pubblico di specialisti, non possono ovviamente risolvere alcun problema nel caso di semplici credenti non avvezzi alle teorie legittimamente proposte e discusse fra i giurisperiti.

Per questo ci pare interessante riportare alcune posizioni emerse circa la questione dei cosiddetti Maqàsid al-shari'a ('finalità della legge islamica') che non da ieri, ma specialmente in epoca contemporanea, stanno avendo particolare fortuna.

Certamente tali riflessioni sono state indotte anche dai rapidi e profondi cambiamenti sociali, ma in quanto rappresentano anche una sorta di 'filosofia del diritto' possono aiutare al superamento di due posizioni estreme e contraddittorie che possiamo anche oggi trovare in rinomati Maestri: da un lato coloro che non si danno troppo pensiero nell'argomentare la validità delle norme poiché ritengono 'giusto' ciò che 
Dio ordina e 'sbagliato' ciò che proibisce, senza troppo indagare, dall'altro quelli che invece si sforzano di trovare anche nuove 'giustificazioni' di talune imposizioni o divieti facendo ricorso anche ad argomentazioni ad esempio igienico-sanitarie che un tempo non andavano di moda, come quando si indicano anche i vantaggi 'fisici' della preghiera islamica, oltre a quelli spirituali, facendo notare che gli inchini e le prostrazioni di cui è composta sarebbero anche un salutare esercizio fisico.

Non è sicuramente cosa nuova in vari tipi di 'modernismi' che pretendono di trovare anticipazioni se non profezie di moderni saperi o persino tecniche nelle fonti antiche ${ }^{21}$, rivelando in questa loro contraddizione una tendenza arcaista soltanto apparentemente contraddittoria con la loro tendenza di fondo.

Resta il fatto che tali ingenuità apologetiche hanno più presa sui comuni credenti che i tecnicismi fin qui riassunti a proposito della verifica dell'autenticità dei detti profetici raccolti nella Sunna.

Permane la classica suddivisione degli atti umani: fard/wājib (obbligatorio); mustahabb/mandūb (raccomandato); mubāh (neutro); makrūh (disapprovato); harām/maḩzūr (proibito).

Naturalmente l'apprezzamento delle loro qualità positive o negative e delle loro conseguenze faceva parte delle motivazioni per cui essi venivano posti nelle differenti categorie.

Anche per quanto riguarda le finalità generali della shari'a (maqasid) esiste una lista di priorità di ciò che esse intendono difendere, mantenere e promuovere: la fede, la vita, la salute, la mente, la prole e l'onore.

È stato rilevato che si tratta di 'valori' più individuali che collettivi, desunti progressivamente più da trattati di giurisperiti che direttamente dalle fonti e che non elencano principi più generali come l'eguaglianza o la libertà. Caratteristiche queste che possono essere rintracciate anche in altri sistemi legali, a seconda dei luoghi e dei tempi. Attualmente la più allargata riflessione sulle finalità della sharia è probabilmente anche una reazione al presente stato di cose: con l'affermazione di movimenti islamici radicali si assiste a una più generalizzata osservanza di determinate regole (ad esempio, il velo femminile); alcuni regimi tentano di legittimarsi religiosamente ricorrendo a punizioni esemplari e tipicamente islamiche (fustigazione per i bevitori, taglio della mano per i ladri, lapidazione per adulterio) e infine le migrazioni in contesti non

21 Cfr. A. DI NOLA, Per una tipologia dei modernismi, in Annali dell'Istituto Universitario Orientale di Napoli, n. 35/2 (1975), pp. 1-8. 
musulmani inducono a privilegiare comportamenti identitari (vestiario, regime alimentare), ma pongono anche altre sfide: è legittimo contrarre un mutuo per acquistare una casa (il prestito a interesse sarebbe vietato dalla sharia), si può lavorare in un locale che offre ai clienti anche alcolici, come comportarsi se la coniuge si converte all'Islam mentre prima non era musulmana?

Va da sé che l'insistenza sul tema dell'identità emerge proprio quando questa è data meno per scontata e si ritiene di difenderla con una più stretta aderenza alle regole, ma è anche da questa stessa crisi che sorgono riflessioni e tentativi di risistemare la materia in modo da collegarla a principi più universalmente riconosciuti e stabili.

Come abbiamo già accennato, proprio la frammentazione del califfato, l'indebolimento e la frammentazione del potere esecutivo, conflitti endemici e un diffuso senso di insicurezza hanno contribuito già nei secoli scorsi a concentrare l'attenzione sul quadro giuridico da preservare come baluardo rispetto a un decadimento generale della Umma.

In tal senso il termine maqasid fu espressamente utilizzato da alJuwayni (m. 1085) che in un'epoca di travaglio e grande confusione immaginava tali finalità e la saggezza in esse intrinseca e da tutti comprensibile potesse essere una garanzia in tempi in cui maestri o governanti fossero deboli, poco affidabili se non del tutto assenti. Il buon senso dell'autore lo spingeva, al contrario di altri, a suggerire per uscire dalla crisi minor intransigenza sui dettagli purché i riti continuassero a svolgersi regolarmente, maggior attenzione alle classi dei diseredati e provvedimenti che facilitassero la ripresa dei commerci.

Anche altri autori affrontarono il problema nelle loro opere, ma quello che poi è rimasto fino a oggi un punto di riferimento fu al-Shatibi (m. 1388), ampiamente citato in una ormai vasta bibliografia ${ }^{22}$.

${ }^{22}$ Cfr. M.T. IBN ASHUR, Treatise on Maqàsid al-Shari'ah, English translation M. ALTAHIR AL-MASAWI, London-Washington, International Institute of Islamic Thought, 2006; A. AL-MALIK AL-JUWAYNI, Ghiàth al-Umam, ed. A.-A. AL-DEEB, Qatar Ministery of Religious Affairs, 1400 AH.; M. IZZI DIEN, Islamic Law: From Historical Foundations to Contemporary Practice, C. HILLENBRAND (ed.), Edinburgh University Press, Edinburgh, 2004); T.J. AL-ALWANI, Maqasid al-Sharìah, Beirut, IIIT 2001; AL-SHATIBI, AlMuwafagàt fì Usùl al-Shari'a, Dawlat Al-Tunisia, 4 volls. (Tunisia), English translation by I.A. NYAZEE.The Reconciliation of the Fundamentals of Islamic Law. Brill 2016; N.H. ABU ZAYD, al-Imàm al-Shàfi'i wa-ta'sìs al-idiùlujiyya al-wasatiyya, Madbuli, Cairo, 2003; E. MOUSSA, The Debts and Burdens of Critical Islam, in Progressive Muslims, O. SAFI (ed.), Oneworld, Oxford 2003; AA. VV., Hiwàr madàsid al-shari'a al-islamiyya, Bayt alHikma, Tunis, 2005. 


\section{1 - Stimoli e riflessioni da un tentativo di approccio comparativo}

All'interno della medesima radice abramica è evidente che sia il Cristianesimo sia l'Islam abbiano conservato parte della precedente religione, ma anche introdotto radicali trasformazioni. Dal punto di vista della normativa si può dire che la frattura fu più decisiva nel caso Ebraismo/Cristianesimo piuttosto che in quello Ebraismo/Islam. Basti pensare che la circoncisione è pratica comune fra i musulmani (e anche fra cristiani orientali) pur non essendo mai nominata nel Corano. L'espansione dell'Islam secondo i paralleli invece che lungo i meridiani può aver favorito tale tendenza, vale a dire che l'attraversamento del Mediterraneo verso nord-ovest da parte del Cristianesimo portò la sua diffusione e istituzionalizzazione progressivamente in ambito grecoromano, da un lato favorendo l'emancipazione dalla legge ebraica e dall'altro spingendo verso il confronto e l'assimilazione col diritto romano e il pensiero greco. Pur mantenendo il legame col Primo Testamento (vivo ancor oggi nella liturgia: si pensi al caso dei salmi) è innegabile la discontinuità coi precetti della Torà. I musulmani non ebbero un'esperienza similare, ricevendo col Corano e poi con la Sunna sia il Testo 'rivelato' ma anche le basi di un sistema giuridico di cui le tribù nomadi arabe erano praticamente prive.

Le similitudini tuttavia non escludono decisive differenze: i molti libri del Primo Testamento sono debitori diretti di una trasmissione orale solo progressivamente scritta, il Secondo Testamento è più limitato in estensione e varietà ed è redatto in greco, il Corano copre un periodo di poco più di vent'anni ed è esclusivamente in arabo. Le prime forme alfabetiche dell'area risalgono a poche migliaia di anni fa, da una radice comune che pur usando segni diversi elencano gli stessi suoni e nello stesso ordine. Ciò non ha comunque impedito una varietà di scritture, di stili e narrazioni che per contenuto e modalità si sono progressivamente fissate in generi letterari, rispondenti alle esigenze e alle finalità dei tempi.

È con la modernità che si sta prospettando per i musulmani qualcosa di simile a quanto è accaduto ai cristiani dei primi secoli, ma in condizioni ben diverse: un nuovo credo ancora flessibile e in fase di vittoriosa espansione nel caso dei secondi, una civiltà uscente da un lungo periodo di decadenza e d'irrigidimento influenzata se non dominata (vedi colonialismo) dall'antico rivale del caso dei primi.

La spinta acculturante occidentale è problematica e sta alla base di molte tensioni, ma la battaglia decisiva si sta combattendo all'interno dell'ecumene musulmana. 
Non si tratta cioè di mantenere o superare ciò che si è ricevuto dalle due precedenti fedi monoteiste, ma di stabilire almeno una gerarchia fra le proprie fonti.

Se finora ci siamo occupati principalmente dell'autorità e del ruolo della Sunna, non mancano posizioni pur isolate ma ancor più estreme rispetto allo stesso Corano.

Abbiamo accennato al movimento dei cosiddetti 'coranisti', secondo i quali il Testo sacro sarebbe sufficiente e gli hadith andrebbero accantonati per la loro scarsa attendibilità, oltre per le contraddizioni che contengono e il loro legame con situazioni obsolete, legate a un ambiente e a un'epoca troppo distanti dalla nostra per poterci dire qualcosa di fondamentale. Il punto debole di questa peraltro sparuta minoranza di studiosi resta quello legato ai dettagli dei riti che senza le specificazioni della Sunna e basandosi solo sul Corano non sarebbero disciplinabili o potrebbero assumere varianti anche di rilievo a seconda delle interpretazioni. Che questa premessa possa potenzialmente condurre a una sorta di 'riforma liturgica' islamica non viene quasi percepito, mentre non ne mancherebbero alcune serie motivazioni: ad esempio, il digiuno di un intero mese dall'alba al tramonto in certi paesi e specie quando il Ramadan cade in estate porta a un rallentamento delle attività che ha un peso economico di cui pochi tengono conto, oppure la concentrazione di milioni di persone alla Mecca per il pellegrinaggio, anch'esso da compiersi esclusivamente in alcuni specifici giorni di un determinato mese, non ha scarse conseguenze sulle strutture ricettive e sugli affollamenti che quasi ogni anno ormai registrano gravi incidenti con un gran numero di vittime

Pur restando tabù questo non secondario tema, c'è stato chi ha tentato di spingersi anche oltre, distinguendo nel Corano stesso ciò che andrebbe ritenuto permanente da quelle che potremmo definire 'disposizioni transitorie' o comunque adattate al momento storico e al luogo e quindi non più vincolanti.

A questo proposito è rilevante il caso del sudanese Abdullahi Ahmed an-Na'im, il quale ha fatto parte del gruppo Republican Brotherhood, fondato dal dotto Mahmud Muhammad Taha, del quale egli stesso ha tradotto in inglese l'opera fondamentale. Già docente all'Università di Khartoum, ha operato in seguito in altri paesi arabi, in Europa e in Nordamerica, dedicandosi in particolare alle problematiche giuridiche e alla questione dei diritti umani. Essendo la Legge una delle espressioni più tipiche del pensiero religioso islamico e avendo essa un impatto diretto sulle tematiche politiche e sociali di più stringente attualità, il contributo di an-Na'im, per quanto risulti per certi aspetti 
piuttosto tecnico e specialistico, è di capitale importanza. Il dibattito a proposito dell'applicazione della sharî́a è, non a caso, uno dei più sentiti sia all'interno del mondo musulmano (dove rappresenta il banco di prova del grado di islamicità non soltanto di pensatori ma anche di movimenti politici e governi) sia al suo esterno quando, a motivo di alcuni casi di libertà di coscienza o di discriminazione tra i sessi, si solleva il problema della compatibilità di determinate disposizioni della legge islamica rispetto a principi e valori cui si ispirano le istituzioni giuridiche internazionali.

Diritto costituzionale, penale, internazionale e diritti umani sono punti caldi che an-Na'im affronta apertamente, senza le ambiguità e le ipocrisie che spesso caratterizzano le prese di posizione ufficiali di molti che si esprimono in proposito, se non con doppiezza, almeno con evidente disagio e poca coerenza. Non si tratta tuttavia sempre e solamente di cattiva volontà. Se molti regimi meritano senz'altro di essere condannati per l'uso pretestuoso che fanno della religione al fine di mantenere individui e gruppi sotto un rigido controllo del tutto insensibile a qualsiasi forma di garanzie democratiche, non si può ignorare una questione di fondo che ha effettivamente una sua rilevanza.

Gli ambiti del diritto or ora menzionati sono stati fortemente influenzati, nei loro fondamenti e metodi, dalla civiltà occidentale. Chiedersi in che modo e fino a che punto essi debbano essere considerati punti di riferimento universali non è dunque una domanda oziosa. Il merito di an-Na'im è quello di lasciarsi interpellare dalle esigenze che stanno alla base di tali aspetti del diritto, cercando una risposta che le soddisfi e, allo stesso tempo, non sia una soluzione extra-islamica. Egli non propone acrobatici sofismi per dimostrare che nel Corano e nella Sunna siano già presenti e garantiti tutti i diritti fondamentali dell'uomo. Riconosce anzi che alcune disposizioni della sharîa, specialmente quelle relative alla condizione femminile e allo statuto dei non-musulmani, sono realmente discriminatori. D'altra parte, essendo un musulmano credente, rispetta il valore normativo delle fonti.

Come uscirne, dunque? La rinuncia all'applicazione dei dettami divini o profetici sarebbe inaccettabile dal punto di vista islamico, ma mantenerli sarebbe incompatibile con le esigenze della modernità. Rifacendosi al pensiero del suo maestro Mahmud Muhammad Taha, egli cerca di superare la 'lettera' di tali norme per coglierne lo 'spirito', ma attraverso un percorso interno alle medesime fonti. In queste ultime, infatti, si trovano anche indicazioni e regole di ben diverso tenore, le quali sono state però in seguito accantonate poiché la primitiva comunità musulmana non era ancora sufficientemente matura per accoglierle. Nulla 
vieta che oggi, a molti secoli di distanza, queste siano recuperate con lo stesso spirito di adattamento alle nuove condizioni concrete della comunità dei credenti, così come in passato - per lo stesso motivo - erano state 'congelate'.

Dal punto di vista della tecnica giuridica islamica si tratta di una vera rivoluzione. Fino a oggi, infatti, è sempre stato osservato il principio in base al quale, nel caso di due versetti coranici contrastanti, è il più tardo a essere considerato valido, cioè abrogante rispetto a quello precedente. In questa nuova visione, invece, avverrebbe esattamente il contrario. E ciò non per un banale desiderio di mettersi al passo coi tempi e compiacere i propri interlocutori occidentali. Ma poiché si riconosce il valore etico universale che sta alla base di determinati principi "la giustificazione etica (di quanto proponiamo) è semplicemente il principio, comune a tutte le tradizioni culturali e religiose del mondo, che si devono trattare gli altri così come si vuole essere trattati da loro", dal quale derivano, ad esempio, la divisione dei poteri, utile a prevenire ingiustizie e abusi. Il fatto che molti paesi dell'Asia e dell'Africa, dopo essersi affrancati dal dominio coloniale, abbiano liberamente adottato sistemi costituzionali dimostrerebbe che si è trattato di una scelta indipendente, fatta sulla base dell'autonomo riconoscimento di un valore e non frutto di un'imposizione. I Testi sacri e l'operato dei Profeti hanno sempre tenuto conto anche delle circostanze, per cui nel Corano - così come nella Bibbia la schiavitù è almeno implicitamente ammessa ... Nessuno, però, potrebbe oggi pretendere di reintrodurla su questa base. Analogamente sarebbe dunque possibile superare quelle forme discriminatorie nei confronti delle donne o dei non musulmani che ancora sussistono. Si tratta di una visione che contestualizza storicamente ciò che molti altri ipostatizzano in forma mitica.

Difficoltà oggettive, comunque, non mancano: un problema spinoso è - ad esempio - quello del superamento delle disposizioni del diritto penale, quando il Corano stesso stabilisce come vanno puniti determinati reati. È pur vero che l'applicazione di tali sanzioni è spesso sottoposta a condizioni quasi impossibili da ottenere (come i quattro testimoni oculari per il reato d'adulterio), ma allo stesso tempo non va dimenticata la discrezionalità con cui si può optare per procedere comunque, soprattutto quando l'autorità voglia legittimarsi su base religiosa decretando fustigazioni, amputazioni di arti o lapidazioni ... Il problema di fondo resterebbe in ogni caso, quand'anche tali pene non fossero in pratica mai applicate, ma sempre teoricamente in vigore. An-Na'im ritiene che la durezza e l'esemplarità di tali punizioni abbiano una loro ratio per il credente, che potrà sempre cercare di convincere anche gli altri della loro 
utilità. In nessun caso, comunque, esse dovrebbero essere imposte ai nonmusulmani contro la loro volontà. Fino a oggi i pensatori musulmani che hanno affrontato la questione dei diritti umani non sono riusciti a proporne una legittimazione culturale fondata sulle stesse fonti del diritto islamico, a parte qualche generica e inconcludente lettura concordistica di taglio meramente apologetico che non ha saputo assumere la centralità della persona e la cura per la sua maturazione come i punti di riferimento principali e come criterio di fondo per la valutazione e l'implementazione di norme e procedure che dovrebbero essere concepite e sviluppate al servizio di tale processo evolutivo.

Pur nei suoi limiti, la proposta di an-Na'im, testimonia invece una tensione morale di alto livello, oltre che offrire interessanti spunti di riflessione e utili sollecitazioni.

\section{2 - Una possibile 'inversione' di tendenza?}

Fin dagli albori l'Islam si è concepito giuridicamente come ordinamento non territoriale, ma di appartenenza religiosa. Già a Medina, subito dopo l'Egira (622) i gruppi islamici ed ebraici della città poterono mantenere il proprio culto e i propri tribunali in base alla fede di appartenenza. Così rimase fino al crollo dei grandi imperi multietnici e pluralistici terminati con la Prima Guerra Mondiale e l'affermarsi dei nazionalismi di stampo europeo illusi di avere stati omogenei per etnia, lingua e religione. Nella Umma islamica, per i musulmani, esistevano doveri individuali (fard 'ayn) come gli atti di culto e doveri collettivi (fard kifàya=obbligo per quella parte sufficiente che doveva compierli, come ad esempio le campagne militari). Dati questi precedenti la situazione migratoria attuale si presenta come una specie di rovesciamento: non dalla Mecca (politeista) a Medina (città di monoteisti), ma il contrario: da una comunità uniforme ad altre pluraliste e persino non religiose. Il dovere individuale inevitabile potrebbe diventare l'interpretazione del Corano, mentre quello collettivo e limitato a un numero sufficiente di credenti quello di mantenere in vita tradizioni ataviche venerande ma non indispensabili, come l'abbigliamento, la capigliatura, i modi di prendere i pasti o di espletare le funzioni fisiologiche sull'esempio del Profeta.

Questo potrebbe significare che un'autentica riforma dell'Islam è più probabile nei territori della diaspora musulmana, dovuta alle recenti e ingenti migrazioni, che non nei paesi d'origine dove molti argomenti rimangono, per dirla con Mohammed Arkoun, 'impensati' se non 'impensabili'. 
È pur vero che episodi di antagonismo estremo, fino ad attacchi terroristici suicidi, sono stati messi in opera da appartenenti alle seconde o terze generazioni approdate in Occidente, ma è altrettanto vero che le opere (in arabo e in altre lingue) su delicate questioni relative all'Islam sono stampati e diffusi quasi solo in Occidente.

Anche per quanto riguarda la fine di un governo centrale della Umma (che comunque il Califfato non è mai stato, essendogli sempre sfuggiti vasti territori musulmani anche nei momenti di massima espansione) la sua fine de iure nel 1924 con la fondazione della Repubblica turca è stata seguita nei primi anni successivi da qualche convegno finalizzato a resuscitarlo, ma poi son sorte organizzazioni panislamiche come la Lega del mondo musulmano o l'Organizzazione della Cooperazione Islamica, paragonabili all'Onu o all'Unesco, che prendono atto del dato di fatto di numerosi e distinti stati chiamati a collaborare più che a fondersi in una velleitaria neo-realtà imperiale, salvo i recenti, maldestri e comunque fallimentari del cosiddetto Isis, supportato anche da volontari musulmani provenienti da ogni dove, ma sostanzialmente limitato a territori arabi del Nordafrica e del Medio Oriente.

Anche la tradizionale ripartizione del mondo in dâr al-Islam (dimora dell'islam, dove i musulmani hanno il potere e/o la maggioranza), dâr alsulh (dimora delle più o meno lunghe 'tregue', prevalentemente con territori confinanti di fede monoteista) e dâr al-jihâd o 'dimora della guerra' (territori pagani da costringere alla conversione, con la non piccola eccezione dell'India, mai maggioritariamente islamizzata) non sembra avere più senso, tanto che specie nella diaspora in Occidente si parla da tempo di dâr al-shahada (dimora della testimonianza) significando che il tempo delle conquiste e riconquiste ha ormai lasciato spazio a territori dove ciascuno manifesta la sua fede che può anche diffondersi ma, per dirla con Papa Francesco, "per attrazione, non per proselitismo".

\section{3 - Con il tempo e con la paglia ...}

Ci sembra evidente che giungere a qualche 'conclusione' sia a questo punto improprio. Non tanto per la comunque sempre valida prudenza che dovrebbe ispirare qualsiasi valutazione in prospettiva storica, né per eclatanti esempi similari in altri contesti: basti pensare che solo il nuovo Codice di Diritto Canonico della Chiesa Cattolica (1983) e 'per delega temporanea' consente alle donne, non il lettorato e il ministero di accolito, ma alcuni ministeri quali: cantori e cantanti, sia come solisti che come 
membri del coro, dirigenti dei servizi liturgici; ministri del battesimo e distributrici dell'Eucarestia.

Lo Stato non pare messo meglio se solo la legge n. 442 del 1981 ha abrogato l'art. 587 del codice penale che puniva il 'delitto d'onore'.

Il fatto è che i processi di mutamento soprattutto antropologici e legati alle questioni di genere in particolare, sono assai lenti e soltanto quando hanno permeato di sé la società possono ambire all'ambito giuridico.

Lo stesso Concilio Vaticano II ha stentato e stenta a esser recepito in molti aspetti, anche se ne abbiamo da poco celebrato il cinquantesimo anniversario e anzi alcune attuali tendenze piuttosto diffuse sembrerebbero indicare un netto arretramento rispetto a molte delle sue affermazioni.

Il tempo, dunque, e 'i tempi' ... ma anche la paglia. Non tanto quella che fa maturare le nespole, come nel noto proverbio, ma quella dell'asino e del bue (non presenti nei Vangeli) tradizionalmente raffigurati attorno alla Sacra neo famiglia di Betlemme. Calore di fiati, pur non di esseri umani, tepore e giusta umidità per un bimbo che soltanto dopo 30 anni avrebbe cominciato la sua missione, e si può ben dire non l'abbia ancora portata a termine almeno per quanto ci è dato di vedere e sperimentare nei limiti del nostro orizzonte terreno. 